\title{
A Versatile Facility for the Calibration of X-ray Polarimeters
}

\author{
Fabio Muleri ${ }^{a, b}$, Paolo Soffitta ${ }^{a}$, Ronaldo Bellazzini ${ }^{c}$, Alessandro Brez ${ }^{c}$, Enrico \\ Costa $^{a}$, Massimo Frutti ${ }^{a}$, Marcello Mastropietro ${ }^{a}$, Massimo Minuti ${ }^{c}$, Ennio Morelli ${ }^{a, d}$, \\ Michele Pinchera $^{c}$, Alda Rubini ${ }^{a}$, Gloria Spandre ${ }^{c}$ \\ ${ }^{a}$ INAF/IASF Rome \\ Via del Fosso del Cavaliere 100, I-00133 Rome, Italy \\ ${ }^{b}$ Università di Roma Tor Vergata, Dipartimento di Fisica \\ via della Ricerca Scientifica 1, 00133 Rome, Italy \\ ${ }^{c}$ INFN Pisa \\ Largo B. Pontecorvo 3, I-56127 Pisa, Italy \\ ${ }^{d}$ INAF/IASF Bologna
}

Via Gobetti 101, I-40129 Bologna, Italy

E-mail: fabio.muleri@iasf-roma.inaf.it

\begin{abstract}
We present a versatile facility built at IASF/INAF of Rome for the calibration of X-ray instruments in the energy range above $1.65 \mathrm{keV}$. Both unpolarized and polarized radiation can be generated. The former is produced by means of radioactive sources or X-ray tubes. Polarized photons are instead obtained by Bragg diffraction at nearly 45 degrees. High fluxes at 2.29, 2.69, 3.69 and 4.51 $\mathrm{keV}$ are produced by diffracting the emission lines of $\mathrm{X}$-ray tubes with molybdenum, rhodium, calcium and titanium anodes on suitable crystals. Diffraction of continuum emission is instead exploited for the production of polarized photons at $1.65 \mathrm{keV}$ and $2.04 \mathrm{keV}$. Moreover it is used to generate polarized photons at higher energies corresponding to the different orders of diffraction. The size and the divergence of beam is controlled with diaphragms and capillary plates. The direction of polarization, the position and the inclination of the beam are accurately controlled by means of high precision motorized stages. This allows to map the response of imaging devices to both polarized and unpolarized radiation. Moreover it is used to study the reponse to inclined beams up to $\sim 60$ degrees.
\end{abstract}

Polarimetry days in Rome: Crab status, theory and prospects October 16-17, 2008

Rome, Italy 


\section{Introduction}

$\mathrm{X}$-ray polarimetry is the last unexplored probe in the X-ray sky. This has been caused by the limited sensitivity of the polarimeters built so far, based on Bragg diffraction and on Thomson scattering. Nevertheless, a wide literature stresses the importance of polarimetry in the study of compact objects and of topics of fundamental physics $[1 ; 2 ; 3 ; 4 ; 5 ; 6]$. Now the development of instruments based on the photoelectric effect provides the possibility to build devices much more sensitive to X-ray polarization [7; 8]. In particular, the Gas Pixel Detector (GPD hereafter) $[7 ; 9 ; 10]$ is one of the most advanced projects in this field.

The calibration of an X-ray polarimeter is not a trivial task. X-ray polarimeters for astrophysical applications have not been developed for many years. Then even their calibration with polarized radiation is not a standard procedure. In the case of the GPD, measurements in the energy range of maximum sensitivity $(2-10 \mathrm{keV})$ has been made possible with the construction of a polarized source based on Bragg diffraction [11].

Here we present a facility designed and built for the systematic calibration of the GPD. This is a further step towards its employement on-board next satellite missions. However we also stress that this facility can be effectively employed even for the calibration of other devices. Imaging detector can exploit the fine positioning of the beam. Spectrometers can be calibrated thanks to the monochromatic beams produced by means of Bragg diffraction [12].

\section{X-ray sources}

Polarized photons are produced with an improved version of the source presented by [13]. Unpolarized photons are generated with an X-ray tube. They are incident at nearly 45 degrees on a crystal. Only the component polarized perpedicularly to the plane of diffraction is effectively reflected. Then the output radition results highly polarized.

The precise degree of polarization $\mathscr{P}$ depends on the diffraction angle $\theta$ :

$$
\mathscr{P}=\frac{1-k(\theta)}{1+k(\theta)} .
$$

$k(\theta)$ is the ratio of the efficiency of diffraction for the components polarized parallel and orthogonally to the plane of diffraction. For $\theta=45^{\circ}, k=0$ and then $\mathscr{P}=1$. Note that the choice of angle $\theta$ selects also the energy of diffracted photons according to the Bragg Law:

$$
E_{B}(\theta)=\frac{n h c}{2 d \sin \theta}
$$

where $h$ and $c$ are respectively Planck's constant and the speed of light, $d$ the crystal lattice spacing and $n$ an integer which specifies the diffraction order.

Note that radiation with a continuum spectrum is diffracted with poor efficiency. A photon with energy $E$ which is incident with an angle $\bar{\theta}$ is diffracted only if $E=E_{B}(\bar{\theta})$. This relation must be satisfied within less than one $\mathrm{eV}$. Only a tiny fraction of the incident radiation is then diffracted. Diffraction is much more effective when monochromatic photons are incident at the corresponding Bragg angle. In this case, about the half of the incident flux is diffracted. 


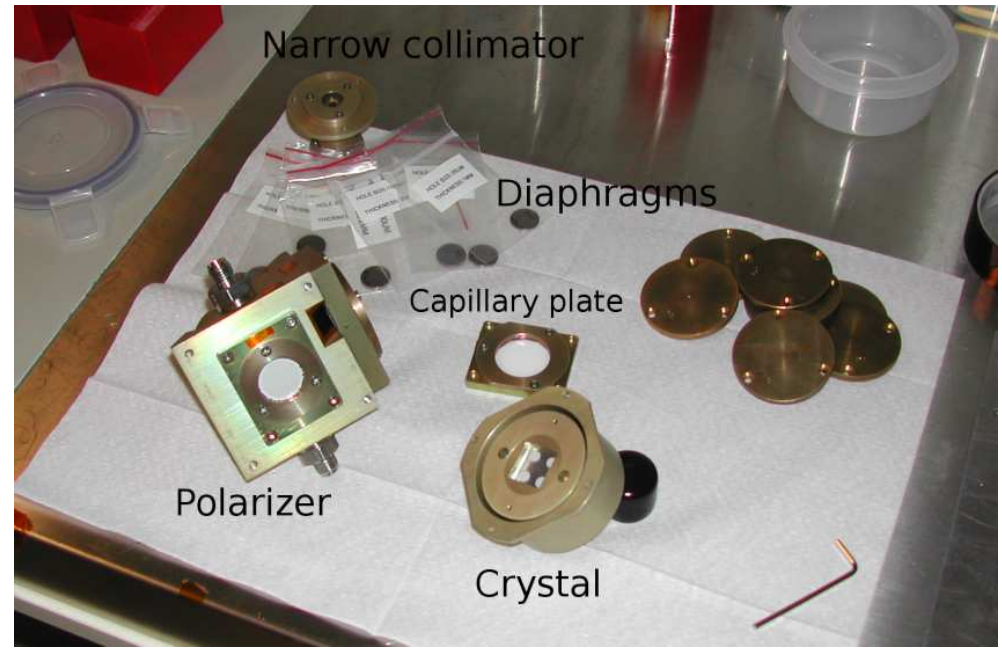

Figure 1: Diaphragms used to control the size of the beam. Their diameter is from $25 \mu \mathrm{m}$ and $2 \mathrm{~mm}$. The very narrow collimator which is obtained with the employment of two diaphragms is on the top left.

A picture of the source is reported in Fig. 1. Capillary plates are employed to constrain the diffraction angle at 45 degrees. The beam can be diaphragmed and collimated by means of capillary plates or even by a narrower collimator. A set of diaphragms from $25 \mu \mathrm{m}$ to $2 \mathrm{~mm}$ can be used to control the size of the beam (see Fig. 1). A very narrow collimator can be constructed by means of two diaphragms separated by a distance of $40 \mathrm{~mm}$. The latter can produce a beam collimated up to a semiaperture $\sim 1 / 1000 \sim 3^{\prime}$ with a size of some tens of $\mu \mathrm{m}$.

In Table 1 we report the polarized lines which can be produce with the Bragg sources. Note that in some cases monochromatic photons are diffracted on suitable crystals at about 45 degrees. In this case the source is particularly effective. In the same Table the energies of the unpolarized radiation which can be produced with X-ray tubes and radioactive sources are also reported.

\section{Mechanical assembly}

The source and the detector are mounted on a mechanical assembly (see Fig. 2(a)). It allows to move, rotate and incline the beam with respect to the detector. Indeed the design of the facility has been driven by three classes of systematic measurements. The first is the mapping of imaging devices, as the GPD. The second is the measurement of the relation between the incident and the reconstructed angle of polarization. Then the plane of polarization must be rotated. Eventually the beam can be inclined to study possible systematic effects. These can be caused for example by the inclined direction of the photons casued by the focusing of X-ray optics.

Eight motorized movements and two manual ones are employed (see Fig. 2(b)). Their characteristics are summarized in Table 2.

\section{Conclusion}

We presented a facility which will be soon used to calibrate the Gas Pixel Detector. Both un- 


\begin{tabular}{|c|c|c|c|c|}
\hline Incident radiation (X-ray tube) & $\mathrm{E}(\mathrm{keV})$ & Crystal & $\theta$ & $\mathscr{P}$ \\
\hline \multicolumn{5}{|c|}{ Polarized radiation } \\
\hline Continuum & 1.65 & $\mathrm{ADP}\left(\mathrm{NH}_{4} \mathrm{H}_{2} \mathrm{PO}_{4}, 101\right)$ & $45^{\circ}$ & $\sim 1.0$ \\
\hline Continuum & 2.04 & $\operatorname{PET}\left(\mathrm{C}\left(\mathrm{CH}_{2} \mathrm{OH}\right)_{4}, 002\right)$ & $45^{\circ}$ & $\sim 1.0$ \\
\hline L $\alpha$ Molybdenum (50 W) & 2.293 & Rhodium (001) & $45.36^{\circ}$ & 0.9994 \\
\hline Continuum & 2.61 & Graphite (002) & $45^{\circ}$ & $\sim 1.0$ \\
\hline L $\alpha$ Rhodium (50 W) & 2.691 & Germanium (111) & $44.86^{\circ}$ & 0.9926 \\
\hline $\mathrm{K} \alpha$ Calcium $(200 \mathrm{~mW})$ & 3.692 & Aluminum (111) & $45.88^{\circ}$ & 0.9938 \\
\hline $\mathrm{K} \alpha$ Titanium $(50 \mathrm{~W})$ & 4.511 & Fluorite $\mathrm{CaF}_{2}(220)$ & $45.37^{\circ}$ & 0.9994 \\
\hline \multicolumn{5}{|c|}{ Unpolarized radiation } \\
\hline L $\alpha$ Molybdenum $(50 \mathrm{~W})$ & 2.293 & - & - & 0 \\
\hline L $\alpha$ Rhodium (50 W) & 2.691 & - & - & 0 \\
\hline $\mathrm{K} \alpha$ Titanium $(50 \mathrm{~W})$ & 4.511 & - & - & 0 \\
\hline $\mathrm{K} \alpha$ Manganese $\left(\mathrm{Fe}^{55}, 5 \mathrm{mCi}\right)$ & 5.899 & - & - & 0 \\
\hline $\mathrm{K} \beta$ Manganese $\left(\mathrm{Fe}^{55}, 5 \mathrm{mCi}\right)$ & 6.490 & - & - & 0 \\
\hline $\mathrm{K} \alpha$ Molybdenum (50 W) & 17.479 & - & - & 0 \\
\hline $\mathrm{K} \alpha$ Rhodium (50 W) & 20.216 & - & - & 0 \\
\hline
\end{tabular}

Table 1: X-ray sources available in the facility. Polarized sources are in the first part of the Table. Continuum radiation is used for diffraction on ADP, PET and graphite crystals. It can be produced by any tube since no line emission in accordance with the Bragg energy at 45 degrees is available. In this case the energy and the polarization in Table are referred to the photons diffracted at 45 degrees. Only the energies corresponding to the first order of diffraction are reported. Note that each crystal can produce polarized radiation even at higher orders. Then energies which are integer multiple of the first order are also available. Polarization from calculation performed by Henke et al.[14]. Unpolarized sources include medium-power X-ray tubes and radioactive sources.

\begin{tabular}{ccccc} 
Name & Range & Resolution & $\begin{array}{c}\text { Load } \\
(\mathrm{kg})\end{array}$ & $\begin{array}{c}\text { Allowed } \\
\text { positions }\end{array}$ \\
\hline \hline$\delta$ & $0 \Leftrightarrow 360^{\circ}$ & $1 ”$ & 20.4 & $0^{\circ} \Leftrightarrow 60^{\circ}, 300^{\circ} \Leftrightarrow 0^{\circ}$ \\
$\varepsilon$ & $0 \Leftrightarrow 360^{\circ}$ & $0.2^{\prime}$ & 183.7 & $0^{\circ} \Leftrightarrow 180^{\circ}, 180^{\circ} \Leftrightarrow 0^{\circ}$ \\
$x$ & $-25 \Leftrightarrow+25 \mathrm{~mm}$ & $0.5 \mu \mathrm{m}$ & 25.5 & $-25 \mathrm{~mm} \Leftrightarrow 25 \mathrm{~mm}$ \\
$y$ & $-25 \Leftrightarrow+25 \mathrm{~mm}$ & $0.5 \mu \mathrm{m}$ & 25.5 & $-25 \mathrm{~mm} \Leftrightarrow 25 \mathrm{~mm}$ \\
$z$ & $0 \Leftrightarrow 50 \mathrm{~mm}$ & - & 10. & $0 \mathrm{~mm} \Leftrightarrow 50 \mathrm{~mm}$ \\
$\eta$ & $0 \Leftrightarrow 25 \mathrm{~mm}$ & $0.1 \mu \mathrm{m}$ & 9.2 & $0 \mathrm{~mm} \Leftrightarrow 25 \mathrm{~mm}$ \\
xso & $0 \Leftrightarrow 25 \mathrm{~mm}$ & $0.1 \mu \mathrm{m}$ & 9.2 & $0 \mathrm{~mm} \Leftrightarrow 25 \mathrm{~mm}$ \\
$y$ so & $0 \Leftrightarrow 25 \mathrm{~mm}$ & $0.1 \mu \mathrm{m}$ & 9.2 & $0 \mathrm{~mm} \Leftrightarrow 25 \mathrm{~mm}$
\end{tabular}

Table 2: Main characteristics of the motorized stages. The load is the maximum allowed on-axis. 


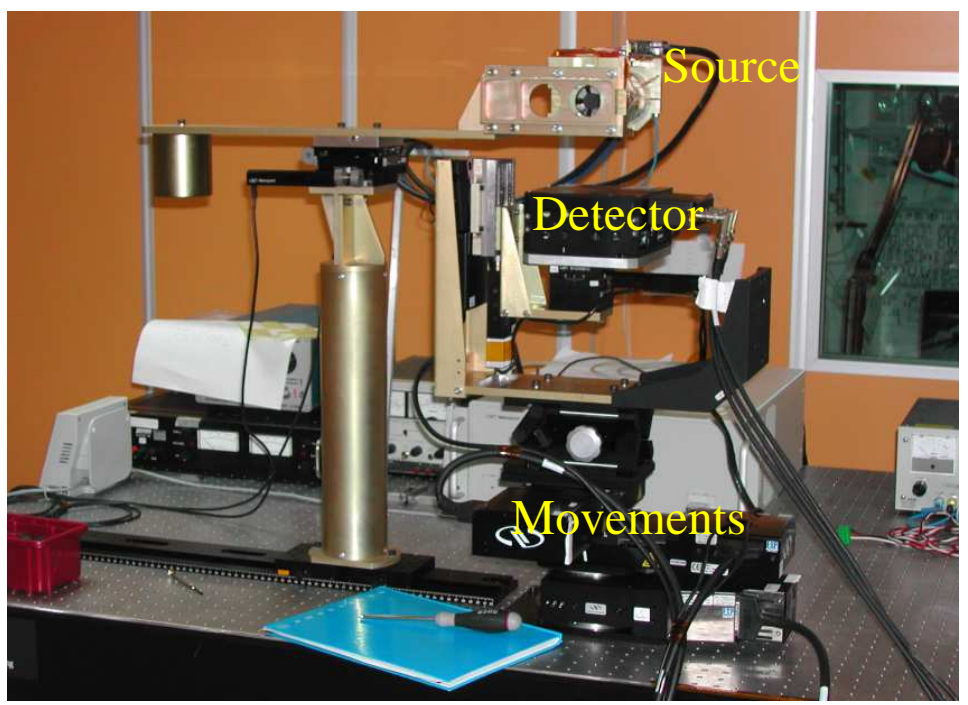

(a)

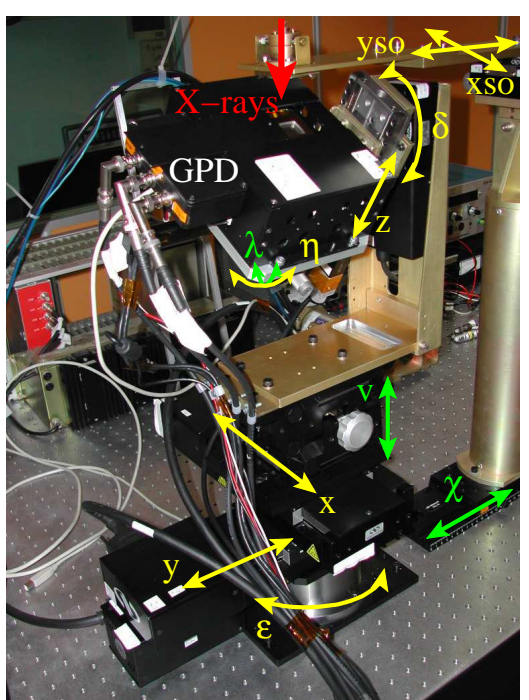

(b)

Figure 2: (a) A picture of the complete facility. The source is on the left and the tower supporting the detector on the right. In this case the unpolarized direct radiation of the titanium anode X-ray tube is employed. (b) The definition of the name of the stages for the towers supporting the detector. In yellow are the motorized stages, while in green are the manual ones.

polarized and polarized radiation can be produced with high fluxes. Collimated and diaphragmed beams can be easily produced. The detector is mounted on a platform which can be moved, rotated and inclined with respect to the beam. This allows to study in detail any possible systematic effect in the measurements of the degree and of the plane of polarization performed with the GPD. Moreover the capability of the facility can also be exploited to study the response of devices dedicated to imaging or spectroscopy.

\section{References}

[1] M. J. Rees. Expected polarization properties of binary X-ray sources. MNRAS, 171:457, 1975.

[2] P. A. Connors, R. F. Stark, and T. Piran. Polarization features of X-ray radiation emitted near black holes. ApJ, 235:224, 1980.

[3] P. Meszaros, R. Novick, A. Szentgyorgyi, G. A. Chanan, and M. C. Weisskopf. Astrophysical implications and observational prospects of X-ray polarimetry. ApJ, 324:1056, 1988.

[4] G. G. Pavlov and V. E. Zavlin. Polarization of Thermal X-Rays from Isolated Neutron Stars. ApJ, 529:1011, 2000.

[5] I. G. Mitrofanov. Astrophysics (communication arising): A constraint on canonical quantum gravity? Nature, 426:139, 2003. 
[6] M. C. Weisskopf, R. F. Elsner, D. Hanna, V. M. Kaspi, S. L. O’Dell, G. G. Pavlov, and B. D. Ramsey. The prospects for X-ray polarimetry and its potential use for understanding neutron stars. Paper Presented at the 363rd Heraeus Seminar in Bad Honef, Germany. Springer Lecture Notes, submitted, 2006.

[7] E. Costa, P. Soffitta, R. Bellazzini, A. Brez, N. Lumb, and G. Spandre. An efficient photoelectric X-ray polarimeter for the study of black holes and neutron stars. Nature, 411:662, 2001.

[8] J. K. Black, R. G. Baker, P. Deines-Jones, J. E. Hill, and K. Jahoda. X-ray polarimetry with a micropattern TPC. Nuclear Instruments and Methods in Physics Research A, 581:755, 2007.

[9] R. Bellazzini, G. Spandre, M. Minuti, L. Baldini, A. Brez, F. Cavalca, L. Latronico, N. Omodei, M. M. Massai, C. Sgro', E. Costa, P. Soffitta, F. Krummenacher, and R. de Oliveira. Direct reading of charge multipliers with a self-triggering CMOS analog chip with $105 \mathrm{k}$ pixels at $50 \mu \mathrm{m}$ pitch. Nuclear Instruments and Methods in Physics Research A, 566:552, 2006.

[10] R. Bellazzini, G. Spandre, M. Minuti, L. Baldini, A. Brez, L. Latronico, N. Omodei, M. Razzano, M. M. Massai, M. Pesce-Rollins, C. Sgró, E. Costa, P. Soffitta, H. Sipila, and E. Lempinen. A sealed Gas Pixel Detector for X-ray astronomy. Nuclear Instruments and Methods in Physics Research A, 579:853, 2007.

[11] F. Muleri, P. Soffitta, L. Baldini, R. Bellazzini, J. Bregeon, A. Brez, E. Costa, M. Frutti, L. Latronico, M. Minuti, M. B. Negri, N. Omodei, M. Pesce-Rollins, M. Pinchera, M. Razzano, A. Rubini, C. Sgró, and G. Spandre. Low energy polarization sensitivity of the Gas Pixel Detector. Nuclear Instruments and Methods in Physics Research A, 584:149, 2008.

[12] M. Feroci, L. Amati, L. A. Antonelli, V. Bonvicini, E. Costa, E. Del Monte, I. Donnarumma, Y. Evangelista, F. Fiore, F. Fuschino, G. L. Israel, G. Labanti, I. Lapshov, F. Lazzarotto, M. Marisaldi, M. Mastropietro, E. Morelli, F. Muleri, F. Nicastro, M. Orlandini, L. Pacciani, F. Perotti, M. Rapisarda, A. Rashevsky, A. Rubini, P. Soffitta, A. Vacchi, G. Zampa, and N. Zampa. A Light and Effective Wide Field Monitor for Gamma Ray Bursts and Transient Sources. In Proc. of The Sixth Huntsville Gamma-Ray Burst Symposium 2008 - October 20-23, 2008.

[13] F. Muleri, P. Soffitta, R. Bellazzini, A. Brez, E. Costa, S. Fabiani, M. Frutti, M. Minuti, M. B. Negri, P. Pascale, A. Rubini, G. Sindoni, and G. Spandre. A very compact polarizer for an X-ray polarimeter calibration. In Proc. of SPIE, volume 6686, page 668610, 2007.

[14] B. L. Henke, E. M. Gullikson, and J. C. Davis. X-Ray Interactions: Photoabsorption, Scattering, Transmission, and Reflection at $\mathrm{E}=50-30,000 \mathrm{eV}, \mathrm{Z}=1-92$. Atomic Data and Nuclear Data Tables, 54:181, 1993. 